Georgian Mathematical Journal

Volume 12 (2005), Number 1, 53-64

\title{
INTERIOR AND EXTERIOR PROBLEMS OF COUPLE-STRESS AND CLASSICAL ELASTOSTATICS WITH GIVEN FRICTION
}

\author{
ROLAND GACHECHILADZE
}

\begin{abstract}
We consider an interior problem of statics of couple-stress elasticity for anisotropic nonhomogeneous media with friction taken into account and the corresponding exterior problem for homogeneous isotropic media. The question of the existence and uniqueness of weak solutions of these problems is studied when friction forces occur along the boundary of an elastic medium or on some part of this boundary.
\end{abstract}

2000 Mathematics Subject Classification: 73T05, 35J85.

Key words and phrases: Friction, couple-stress elasticity, statics, boundary variational inequality, anisotropic nonhomogeneous medium.

In the present paper we consider deformation of an elastic medium with friction conditions imposed all along its boundary or on some part of the boundary described by Coulomb's law. Problems of this kind for bounded domains are studied mainly in [1]-[6], where these problems are reduced to a variational inequality in the domain and, after that, the existence and uniqueness of a solution of this inequality are investigated.

In our case, the considered problems are equivalently reduced to a variational inequality not in the domain, but on the boundary of an elastic medium. Boundary variational inequalities make it possible to investigate exterior problems with friction and are convenient for obtaining approximate solutions. We begin our consideration with interior problems.

\section{INTERIOR PROBLEMS}

Let $\Omega^{+} \subset \mathbb{R}^{3}$ be the bounded domain with boundary $\Gamma\left(\Gamma \in C^{\infty}\right), \Omega^{-}=\mathbb{R}^{3} \backslash \bar{\Omega}^{+}$, $\nu(x)$ be the exterior normal unit vector (with respect to $\Omega^{ \pm}$) at a point $x \in \Gamma$;

$$
\mathcal{M}(x, \partial)=\left\|\begin{array}{l:c}
\mathcal{M}^{(1)}(x, \partial) & \mathcal{M}^{(2)}(x, \partial) \\
----- & ----- \\
\mathcal{M}^{(3)}(x, \partial) & \mathcal{M}^{(4)}(x, \partial)
\end{array}\right\|_{6 \times 6}
$$

be a matrix differential operator of statics of couple-stress elasticity, and

$$
\mathcal{N}(x, \partial, \nu)=\left\|\begin{array}{l:c}
\mathcal{N}^{(1)}(x, \partial, \nu) & \mathcal{N}^{(2)}(x, \partial, \nu) \\
------ & ------ \\
\mathcal{N}^{(3)}(x, \partial, \nu) & \mathcal{N}^{(4)}(x, \partial, \nu)
\end{array}\right\|_{6 \times 6}
$$


be a stress operator (the operators $\mathcal{M}^{(j)}(x, \partial)=\left\|\mathcal{M}_{l m}^{(j)}(x, \partial)\right\|_{3 \times 3}$ and $\mathcal{N}^{(j)}(x, \partial, \nu)=\left\|\mathcal{N}_{l m}^{(j)}(x, \partial, \nu)\right\|_{3 \times 3}, j=1,2,3,4$, are defined in [4], [7]); $U=(u, \omega), u=\left(u_{1}, u_{2}, u_{3}\right)$ be a shifting vector, $\omega=\left(\omega_{1}, \omega_{2}, \omega_{3}\right)$ be a rotation vector, $\sigma(U)=\mathcal{N}^{(1)}(x, \partial, \nu) u(x)+\mathcal{N}^{(2)}(x, \partial, \nu) \omega(x)$ be a force vector, $\mu(U)=\mathcal{N}^{(3)}(x, \partial, \nu) u(x)+\mathcal{N}^{(4)}(x, \partial, \nu) \omega(x)$ be a couple-stress vector; $a_{T}$ and $a_{N}$ denote respectively tangential and normal components of a vector $a \in \mathbb{R}^{3}$ (with respect to $\Omega^{+}$).

The real functions $a_{i j e k}, b_{i j e k}, c_{i j e k}$, participating in the definition of the operator $\mathcal{M}(x, \partial)$ (physical characteristics of an elastic body) satisfy the following conditions:

(i) $a_{i j e k}, b_{i j e k}, c_{i j e k} \in C^{\infty}\left(\bar{\Omega}^{+}\right)$;

(ii) $a_{i j e k}=a_{e k i j}, c_{i j e k}=c_{e k i j}$;

(iii) $\exists \alpha_{0}>0$ be such that $\forall x \in \bar{\Omega}^{+}$and $\forall \xi_{i j}, \eta_{i j} \in \mathbb{R}$ :

$$
a_{i j e k}(x) \xi_{i j} \xi_{e k}+2 b_{i j e k}(x) \eta_{e k} \xi_{i j}+c_{i j e k}(x) \eta_{i j} \eta_{e k} \geq \alpha_{0}\left(\xi_{i j} \xi_{i j}+\eta_{i j} \eta_{i j}\right)
$$

(here and in what follows the repetition of the index means summation over this index from 1 to 3$)$.

We denote by $H^{s}\left(\Omega^{+}\right), H_{\text {loc }}^{s}\left(\Omega^{-}\right)$and $H^{s}(\Gamma)$ the Sobolev-Slobodetski space for $s \in \mathbb{R}([8])$ and assume that $w \in X^{m}$ if each component of the vector $w=\left(w_{1}, \ldots, w_{m}\right)$ belongs to some space $X$.

For each $U=(u, \omega) \in\left(H^{1}\left(\Omega^{+}\right)\right)^{6}$ and $V=(v, w) \in\left(H^{1}\left(\Omega^{+}\right)\right)^{6}$ we define the bilinear form $\mathcal{B}(U, V)$ as

$$
\begin{aligned}
\mathcal{B}(U, V)= & \int_{\Omega^{+}}\left[a_{i j e k}(x) \xi_{i j}(U) \xi_{e k}(V)+b_{i j e k}(x) \xi_{i j}(U) \eta_{e k}(V)\right. \\
& \left.+b_{e k i j}(x) \xi_{i j}(V) \eta_{e k}(U)+c_{i j e k}(x) \eta_{i j}(U) \eta_{e k}(V)\right] d x
\end{aligned}
$$

where $\xi_{i j}(U)=\frac{\partial u_{j}}{\partial x_{i}}-\varepsilon_{i j k} \omega_{k}, \eta_{i j}(U)=\frac{\partial \omega_{j}}{\partial x_{i}}$, and $\varepsilon_{i j k}$ is the Levi-Civita symbol.

Definition. A vector function $U \in\left(H^{1}\left(\Omega^{+}\right)\right)^{6}$ is a weak solution of the equation

$$
\mathcal{M}(x, \partial) U(x)+\mathcal{G}(x)=0 \quad\left(\mathcal{G} \in\left(L^{2}\left(\Omega^{+}\right)\right)^{6}\right)
$$

if $\forall \Phi \in\left(C_{0}^{\infty}\left(\Omega^{+}\right)\right)^{6}$

$$
\mathcal{B}(U, \Phi)=(\mathcal{G}, \Phi)_{0, \Omega^{+}} \quad\left((\varphi, \psi)_{0, \Omega^{+}}=\int_{\Omega^{+}} \varphi \bar{\psi} d x\right) .
$$

Note that if $U \in\left(H^{1}\left(\Omega^{+}\right)\right)^{6}$ and $\mathcal{M} U \in\left(L^{2}\left(\Omega^{+}\right)\right)^{6}$, then $\left.\mathcal{N}(x, \partial, \nu) U(x)\right|_{\Gamma}$ can be defined as a functional of the class $\left(H^{-1 / 2}(\Gamma)\right)^{6}$ by means of the formula

$$
\left\langle\left.\mathcal{N}(x, \partial, \nu) U(x)\right|_{\Gamma}, \Phi\right\rangle=(\mathcal{M} U, V)_{0, \Omega^{+}}+\mathcal{B}(U, V), \quad \forall \Phi \in\left(H^{1 / 2}(\Gamma)\right)^{6}
$$

and $\forall V \in\left(H^{1}\left(\Omega^{+}\right)\right)^{6},\left.V\right|_{\Gamma}=\Phi$. Here the brackets $\langle\cdot, \cdot\rangle$ mean a relation of duality between the dual pairs $\left(H^{-1 / 2}(\Gamma)\right)^{6}$ and $\left(H^{1 / 2}(\Gamma)\right)^{6}$. 
Let $\Gamma=\Gamma_{1} \cup \Gamma_{2}, \Gamma_{1} \cap \Gamma_{2}=\varnothing$ and $\mathcal{G} \in\left(L^{2}\left(\Omega^{+}\right)\right)^{6}, \chi \in\left(H^{1 / 2}\left(\Gamma_{1}\right)\right)^{6}, F_{N} \in$ $L^{\infty}\left(\Gamma_{2}\right), \psi \in\left(L^{\infty}\left(\Gamma_{2}\right)\right)^{3}, \mathcal{F}: \Gamma_{2} \rightarrow \mathbb{R}, \mathcal{F} \in L^{\infty}\left(\Gamma_{2}\right), \mathcal{F} \geq 0, g=\mathcal{F}\left|F_{N}\right|$.

Problem $(\mathbf{I})^{+}$. Find a vector function $U \in\left(H^{1}\left(\Omega^{+}\right)\right)^{6}$ which is a weak solution of equation (2), tangential component of force on $\Gamma_{2}$ is a function of the class $\left(L^{\infty}\left(\Gamma_{2}\right)\right)^{3}$ and the following conditions are fulfilled:

$$
\left.U\right|_{\Gamma_{1}}=\chi,\left.\quad \mu U\right|_{\Gamma_{2}}=\psi,\left.\quad \sigma_{N}(U)\right|_{\Gamma_{2}}=F_{N} \text { and if }\left|\sigma_{T}(U)\right|_{\Gamma_{2}}<g
$$

then $u_{T}=0$, and if $\left|\sigma_{T}(U)\right|_{\Gamma_{2}}=g$, then $\exists \lambda \geq 0: u_{T}=-\lambda \sigma_{T}(U)$.

Let $U_{0}=\left(u_{0}, \omega_{0}\right) \in\left(H^{1}\left(\Omega^{+}\right)\right)^{6}$ be a weak solution of equation (2) which satisfies the conditions $\left.U_{0}\right|_{\Gamma_{1}}=\chi$ and $\left.\mathcal{N} U\right|_{\Gamma_{2}}=0$ (as is known, this problem has a unique solution). Then for the vector function $V=U-U_{0}$ (instead of which we write $U$ ) we obtain

Problem $(\mathbf{F})^{+}$. Find a vector function $U \in\left(H^{1}\left(\Omega^{+}\right)\right)^{6}$ which is a weak solution of the equation

$$
\mathcal{M}(x, \partial) U(x)=0
$$

and satisfies the conditions

$$
\begin{aligned}
& \left.\sigma_{T}(U)\right|_{\Gamma_{2}} \in\left(L^{\infty}\left(\Gamma_{2}\right)\right)^{3},\left.\quad U\right|_{\Gamma_{1}}=0,\left.\mu U\right|_{\Gamma_{2}}=\psi,\left.\quad \sigma_{N}(U)\right|_{\Gamma_{2}}=F_{N} \text { and if } \\
& \left|\sigma_{T}(U)\right|_{\Gamma_{2}}<g \text {, then } u_{T}=\varphi_{T} \text {, and if }\left|\sigma_{T}(U)\right|_{\Gamma_{2}}=g \text {, then } \exists \lambda \geq 0 \text { : } \\
& u_{T}=\varphi_{T}-\lambda \sigma_{T}(U), \\
& \text { where } \varphi_{T}=-\left.u_{0 T}\right|_{\Gamma} \in\left(H^{1 / 2}(\Gamma)\right)^{3} \text {. }
\end{aligned}
$$

Let us reduce Problem $(F)^{+}$to a boundary variational inequality.

Let $G^{+}:\left(H^{1 / 2}(\Gamma)\right)^{6} \longrightarrow\left(H^{1}\left(\Omega^{+}\right)\right)^{6}$ be the Green operator of the Dirichlet problem, i.e., $\forall h \in\left(H^{1 / 2}(\Gamma)\right)^{6}, G^{+} h \in\left(H^{1}\left(\Omega^{+}\right)\right)^{6}$ is a weak solution of equation (3) and $\left.G^{+} h\right|_{\Gamma}=h$ (it is easy to prove the existence and uniqueness of such a solution). By virtue of the trace operator and by applying the Banach theorem we can prove that there exist constants $c_{i}>0, i=1,2$, such that $\forall h \in\left(H^{1 / 2}(\Gamma)\right)^{6}$ :

$$
c_{1}\|h\|_{1 / 2, \Gamma} \leq\left\|G^{+} h\right\|_{1, \Omega^{+}} \leq c_{2}\|h\|_{1 / 2, \Gamma} .
$$

Define the operator $L^{+}:\left(H^{1 / 2}(\Gamma)\right)^{6} \longrightarrow\left(H^{-1 / 2}(\Gamma)\right)^{6}$ by the formula

$$
L^{+} h=\left\{\mathcal{N}(x, \partial, \nu)\left(G^{+} h\right)(x)\right\}_{\Gamma}^{+}, \quad \forall h \in\left(H^{1 / 2}(\Gamma)\right)^{6} .
$$

It should be noted that the definition of the operator $L^{+}$is correct since $G^{+} h \in\left(H^{1}\left(\Omega^{+}\right)\right)^{6}$ and $\mathcal{M}\left(G^{+} h\right)=0 \in\left(L^{2}\left(\Omega^{+}\right)\right)^{6}$.

The following properties are valid for the operator $L^{+}$:

(I) $\left\langle L^{+} h, g\right\rangle=\left\langle L^{+} g, h\right\rangle, \forall h, g \in\left(H^{1 / 2}\left(\Omega^{+}\right)\right)^{6}$;

(II) $L^{+}:\left(H^{1 / 2}(\Gamma)\right)^{6} \longrightarrow\left(H^{-1 / 2}(\Gamma)\right)^{6}$ is a continuous mapping;

(III) $\exists c>0:\left\langle L^{+} h, h\right\rangle \geq c\|P h\|_{1 / 2, \Gamma}^{2}, \quad \forall h \in\left(H^{1 / 2}(\Gamma)\right)^{6}$;

(IV) $\exists c>0:\left\langle L^{+} h, h\right\rangle \geq c\|h\|_{1 / 2, \Gamma}^{2}, \forall h \in\left(H^{1 / 2}(\Gamma)\right)^{6},\left.\quad h\right|_{\Gamma_{1}}=0$, 
where $I-P$ is the operator of orthogonal projection of the space $\left(H^{1 / 2}(\Gamma)\right)^{6}$ (in a sense of the space $H^{1 / 2}$ ) onto the set of solution of the equation $\left\langle L^{+} h, h\right\rangle=0$ (the space of solutions of this equation coincides with the set of traces on the boundary $\Gamma$ of rigid displacement vectors).

Properties (I) and (II) of the operator $L^{+}$follow from the continuity of the bilinear form (1) and inequality (4). We will prove (III). Applying Lemma 1 ([4]), we have

$$
\left\langle L^{+} h, h\right\rangle=\mathcal{B}\left(G^{+} h, G^{+} h\right) \geq c\left\|P_{1}\left(G^{+} h\right)\right\|_{1, \Omega^{+}}^{2}, \quad \forall h \in\left(H^{1 / 2}(\Gamma)\right)^{6},
$$

where $I-P_{1}$ is the operator of orthogonal projection of the space $\left(H^{1}\left(\Omega^{+}\right)\right)^{6}$ onto the set of rigid displacement vectors $\mathcal{R}\left(\mathcal{R}=\left\{U=(u, \omega) \in\left(H^{1}\left(\Omega^{+}\right)\right)^{6}\right.\right.$ : $\left.\left.u=[a \times x]+b, \omega=a, a, b \in \mathbb{R}^{3}\right\}\right)$, i.e., $\exists U_{h} \in \mathcal{R}$ such that

$$
P_{1}\left(G^{+} h\right)=G^{+} h-U_{h} .
$$

In view of inequality (4) we obtain

$$
\begin{aligned}
\left\|P_{1}\left(G^{+} h\right)\right\|_{1, \Omega^{+}} & =\left\|G^{+} h-G^{+}\left(\left.U_{h}\right|_{\Gamma}\right)\right\|_{1, \Omega^{+}}=\left\|G^{+}\left(h-\left.U_{h}\right|_{\Gamma}\right)\right\|_{1, \Omega^{+}} \\
& \geq c_{1}\left\|h-\left.U_{h}\right|_{\Gamma}\right\|_{1 / 2, \Gamma} \geq c\|P h\|_{1 / 2, \Gamma}, \quad \forall h \in\left(H^{1 / 2}(\Gamma)\right)^{6} .
\end{aligned}
$$

Thus (III) is proved. Property (IV) is a simple corollary of (III).

Assume that $\mathcal{K}=\left\{h=(\xi, \eta) \in\left(H^{1 / 2}(\Gamma)\right)^{6}:\left.h\right|_{\Gamma_{1}}=0\right\}$ and consider, on the space $\left(H^{1 / 2}(\Gamma)\right)^{3}$, the continuous convex functional

$$
j(\xi)=\int_{\Gamma_{2}} g\left|\xi_{T}-\varphi_{T}\right| d s
$$

and the following variational inequality: find $h_{0}=\left(\xi_{0}, \eta_{0}\right) \in \mathcal{K}$ such that $\forall h=$ $(\xi, \eta) \in \mathcal{K}:$

$$
\left\langle L^{+} h_{0}, h-h_{0}\right\rangle+j(\xi)-j\left(\xi_{0}\right) \geq \int_{\Gamma_{2}}\left[F_{N}\left(\xi_{N}-\xi_{0 N}\right)+\psi \cdot\left(\eta-\eta_{0}\right)\right] d s .
$$

Theorem 1. The boundary variational inequality (5) and problem $(F)^{+}$are equivalent.

Proof. It should be noted that the equivalence is understood in the following sense: if $U \in\left(H^{1}\left(\Omega^{+}\right)\right)^{6}$ is a solution of Problem $(F)^{+}$, then $\left.U\right|_{\Gamma}=h_{0}$ is a solution of inequality (5) and, conversely, if $h_{0} \in \mathcal{K}$ is a solution of inequality (5), then $G^{+} h \in\left(H^{1}\left(\Omega^{+}\right)\right)^{6}$ is a solution of Problem $(F)^{+}$.

Let $U \in\left(H^{1}\left(\Omega^{+}\right)\right)^{6}$ be a solution of Problem $(F)^{+}$and $\left.U\right|_{\Gamma}=h_{0}$ (clearly, by virtue of the definition of the Green operator, $\left.U=G^{+} h_{0}\right)$.

One can easily verify that if the conditions of Problem $(F)^{+}$are fulfilled, then the inequality

$$
\sigma_{T}\left(G^{+} h_{0}\right) \cdot\left(\xi_{T}-\xi_{0 T}\right)+g\left(\left|\xi_{T}-\varphi_{T}\right|-\left|\xi_{0 T}-\varphi_{T}\right|\right) \geq 0
$$


holds on $\Gamma_{2}$. After integrating (6) on $\Gamma_{2}$, we obtain

$$
\begin{aligned}
& \int_{\Gamma_{2}} \sigma_{T}\left(G^{+} h_{0}\right) \cdot\left(\xi_{T}-\xi_{0 T}\right) d s+\int_{\Gamma_{2}} \mu\left(G^{+} h_{0}\right) \cdot\left(\eta-\eta_{0}\right) d s \\
& \quad+\int_{\Gamma_{2}} \sigma_{N}\left(G^{+} h_{0}\right)\left(\xi_{N}-\xi_{0 N}\right) d s+j(\xi)-j\left(\xi_{0}\right) \\
& \geq \int_{\Gamma_{0}}\left[\sigma_{N}\left(G^{+} h_{0}\right)\left(\xi_{N}-\xi_{0 N}\right)+\mu\left(G^{+} h_{0}\right) \cdot\left(\eta-\eta_{0}\right)\right] d s,
\end{aligned}
$$

i.e., (5) is fulfilled.

Conversely, let $h_{0} \in \mathcal{K}$ be a solution of inequality (5). By virtue of the definition of the Green operator, $U=G^{+} h_{0}$ is a weak solution of equation (3) and $\left.U\right|_{\Gamma_{1}}=\left.G^{+} h_{0}\right|_{\Gamma_{1}}=\left.h_{0}\right|_{\Gamma_{1}}=0$ since $h_{0} \in \mathcal{K}$.

Let $h \in \mathcal{K}$ be such that $\xi_{T}=\xi_{0 T}, \eta=\eta_{0}$, and $\xi_{N}=\xi_{0 N} \pm \Theta$, where $\Theta \in H^{1 / 2}(\Gamma), \operatorname{supp} \Theta \subset \Gamma_{2}$. Then $j(\xi)=j\left(\xi_{0}\right)$ and from (5) it follows that

$$
\left\langle\sigma_{N}\left(G^{+} h_{0}\right), \Theta\right\rangle=\int_{\Gamma_{2}} F_{N} \Theta d s, \forall \Theta \in H^{1 / 2}(\Gamma), \quad \operatorname{supp} \Theta \subset \Gamma_{2} .
$$

Therefore

$$
\left.\sigma_{N}\left(G^{+} h_{0}\right)\right|_{\Gamma_{2}}=F_{N}
$$

Analogously, choosing $h \in \mathcal{K}$ in an appropriate manner, we obtain

$$
\left.\mu\left(G^{+} h_{0}\right)\right|_{\Gamma_{2}}=\psi
$$

In view of (7) and (8), inequality (5) can be rewritten as

$$
\int_{\Gamma_{2}}\left[\sigma_{T}\left(G^{+} h_{0}\right) \cdot \chi_{T}+g\left|\chi_{T}\right|\right] d s-\int_{\Gamma_{2}}\left[\sigma_{T}\left(G^{+} h_{0}\right) \cdot \chi_{0 T}+g\left|\chi_{0 T}\right|\right] d s \geq 0,
$$

where $\chi_{T}=\xi_{T}-\varphi_{T}$ and $\chi_{0 T}=\xi_{0 T}-\varphi_{T}$. Let

$$
\Psi=\left\{\zeta \in\left(H^{1 / 2}(\Gamma)\right)^{3}:\left.\zeta\right|_{\Gamma_{1}}=0\right\} .
$$

Replacing $\chi_{T}$ in (9) by $\chi_{0 T} \pm \zeta_{T}$, where $\zeta \in \Psi$, and taking into account that $\left|\zeta_{T}\right| \leq|\zeta|$, after some transformations we obtain

$$
\left|\int_{\Gamma_{2}} \sigma_{T}\left(G^{+} h_{0}\right) \cdot \zeta d s\right| \leq \int_{\Gamma_{2}} g|\zeta| d s, \quad \forall \zeta \in \Psi
$$

and

$$
\int_{\Gamma_{2}}\left[\sigma_{T}\left(G^{+} h_{0}\right) \cdot \chi_{0 T}+g\left|\chi_{0 T}\right|\right] d s \leq 0 .
$$

Let us consider the functional

$$
\Phi(\zeta)=\int_{\Gamma_{2}} \sigma_{T}\left(G^{+} h_{0}\right) \cdot \zeta d s, \quad \zeta \in \Psi
$$


on the set $\Psi$.

By virtue of (10) the functional $\Phi$ on the space $\Psi \subset\left(L^{1}(\Gamma)\right)^{3}$ is linear and continuous in the induced topology and its norm does not exceed one.

Since $\left.\Psi\right|_{\Gamma_{2}}$ is dense in $\left(L^{1}\left(\Gamma_{2}\right)\right)^{3}$, by virtue of the Hahn-Banach theorem $\Phi \in\left(L^{\infty}\left(\Gamma_{2}\right)\right)^{3}$ and $\|\Phi\| \leq 1$, i.e.,

$$
\sigma_{T}\left(G^{+} h_{0}\right) \in\left(L^{\infty}\left(\Gamma_{2}\right)\right)^{3} \text {. }
$$

Let us write the functional $\Phi$ in a different form:

$$
\Phi(\zeta)=\int_{\Gamma_{2}} g^{-1} \sigma_{T}\left(G^{+} h_{0}\right) \cdot g \zeta d s
$$

(assuming that $g \geq g_{0}>0$ ).

Applying our preceding arguments to functional (12), we obtain

$$
\left|\sigma_{T}\left(G^{+} h_{0}\right)\right| \leq g .
$$

In view of (13), from (11) we have

$$
\sigma_{T}\left(G^{+} h_{0}\right) \cdot \chi_{0 T}+g\left|\chi_{0 T}\right|=0,
$$

which in turn ensures the fulfilment of the friction condition of Problem $(F)^{+}$.

Let us investigate the question of existence and uniqueness of a solution of inequality (5).

Let mes $\Gamma_{1}>0$. On the convex closed set $\mathcal{K}$ we consider the functional

$$
I(h)=\frac{1}{2}\left\langle L^{+} h, h\right\rangle+j(\xi)-\int_{\Gamma_{2}}\left[F_{N} \xi_{N}+\psi \cdot \eta\right] d s, \quad \forall h=(\xi, \eta) \in \mathcal{K} .
$$

One can easily easily verify that by virtue of property (I) of the operator $L^{+}$, the solution of inequality (5) is equivalent to minimization of the functional $I(h)$ on the set $\mathcal{K}$.

Taking into account property (IV) of the operator $L^{+}$and the fact that $j(\xi) \geq 0$, we obtain

$$
I(h) \geq c\|h\|_{1 / 2, \Gamma}^{2}-c_{1}\|h\|_{1 / 2, \Gamma}, \quad \forall h \in \mathcal{K},
$$

i.e., $I(h) \longrightarrow+\infty$ as $\|h\|_{1 / 2, \Gamma} \longrightarrow \infty$. This means that the functional $I(h)$ is coercive and, on the basis of the known results (see [9], [10]), we conclude that Problem $(F)^{+}$has a unique solution. Thus we arrive at

Theorem 2. If $\mathcal{G} \in\left(L^{2}\left(\Omega^{+}\right)\right)^{6}, F_{N} \in L^{\infty}\left(\Gamma_{2}\right)$ and $\psi \in\left(L^{\infty}\left(\Gamma_{2}\right)\right)^{3}$, then Problem $(F)^{+}$has a unique solution of the class $\left(H^{1}\left(\Omega^{+}\right)\right)^{6}$.

Let now $\Gamma_{1}=\varnothing$. Then $\Gamma_{2}=\Gamma$ and instead of Problem $(\mathrm{I})^{+}$we consider

Problem $(\mathbf{I I})^{+}$. Assume that $\mathcal{G} \in\left(L^{2}\left(\Omega^{+}\right)\right)^{6}, F_{N} \in L^{\infty}(\Gamma), \psi \in\left(L^{\infty}(\Gamma)\right)^{3}$, $\mathcal{F} \in L^{\infty}(\Gamma), \mathcal{F} \geq 0$ and $g=\mathcal{F}\left|F_{N}\right|$. Find a vector function $U=(u, \omega) \in$ 
$\left(H^{1}\left(\Omega^{+}\right)\right)^{6}$ which is a weak solution of equation (2) and satisfies the following conditions:

$$
\begin{aligned}
& \left.\sigma_{T}(U)\right|_{\Gamma} \in\left(L^{\infty}(\Gamma)\right)^{3},\left.\mu(U)\right|_{\Gamma}=\psi,\left.\sigma_{N}(U)\right|_{\Gamma}=F_{N}, \text { and if }\left|\sigma_{T}(U)\right|_{\Gamma}<g, \\
& \text { then } u_{T}=0 \text {, and if }\left|\sigma_{T}(U)\right|_{\Gamma}=g, \text { then } \exists \lambda \geq 0: u_{T}=-\lambda \sigma_{T}(U) .
\end{aligned}
$$

Let $\Omega^{+}$be not a body of rotation, and $U_{0}=\left(u_{0}, \omega_{0}\right) \in\left(H^{1}\left(\Omega^{+}\right)^{6}\right.$ be a weak solution of equation (2) for which

$$
\left.u_{0 N}\right|_{\Gamma}=0,\left.\quad \sigma_{T}\left(U_{0}\right)\right|_{\Gamma}=0 \text { and }\left.\mu\left(U_{0}\right)\right|_{\Gamma}=0
$$

(as is known, there exists a unique solution of this problem).

Then for the vector function $V=U-U_{0}$ (instead of which, like previously, we again write $U$ ) we obtain

Problem $(\Psi)^{+}$. Find a vector function $U=(u, \omega) \in\left(H^{1}\left(\Omega^{+}\right)\right)^{6}$ which is a weak solution of equation (3) and satisfies, on the boundary $\Gamma$, the following conditions:

$$
\begin{aligned}
& \sigma_{T}(U) \in\left(L^{\infty}(\Gamma)\right)^{3}, \sigma_{N}(U)=\Psi_{N}, \mu(U)=\psi \text { and if }\left|\sigma_{T}(U)\right|<g \text {, then } \\
& u_{T}=\varphi_{T}, \text { and if }\left|\sigma_{T}(U)\right|=g \text {, then } \exists \lambda \geq 0: u_{T}=\varphi_{T}-\lambda \sigma_{T}(U) \text {, where } \\
& \Psi_{N}=F_{N}-\sigma_{N}\left(U_{0}\right) \text { and } \varphi_{T}=-u_{0 T} .
\end{aligned}
$$

As before, Problem $(\Psi)^{+}$and the following variational inequality are equivalent (the equivalence is understood as in Theorem 1 ):

Find $h_{0}=\left(\xi_{0}, \eta_{0}\right) \in\left(H^{1 / 2}(\Gamma)\right)^{6}$ such that $\forall h=(\xi, \eta) \in\left(H^{1 / 2}(\Gamma)\right)^{6}$

$$
\left\langle L^{+} h_{0}, h-h_{0}\right\rangle+j(\xi)-j\left(\xi_{0}\right) \geq \int_{\Gamma}\left[\Psi_{N}\left(\xi_{N}-\xi_{0 N}\right)+\psi \cdot\left(\eta-\eta_{0}\right)\right] d s,
$$

where

$$
j(\xi)=\int_{\Gamma} g\left|\xi_{T}-\varphi_{T}\right| d s .
$$

Let $h_{0} \in\left(H^{1 / 2}(\Gamma)\right)^{6}$ be a solution of inequality (14). Substituting into (14) first $h=\left(\varphi_{T}, \eta_{0}\right)$ and then $h=\left(2 \xi_{0}-\varphi_{T}, \eta_{0}\right)$ instead of $h=(\xi, \eta)$, we obtain

$$
\left\langle\sigma\left(G^{+} h_{0}\right), \xi_{0}-\varphi_{T}\right\rangle+j\left(\xi_{0}\right)=\int_{\Gamma} \Psi_{N} \xi_{0 N} d s .
$$

Taking into account (15), from (14) we have

$$
\begin{gathered}
\left\langle\sigma\left(G^{+} h_{0}\right), \xi-\varphi_{T}\right\rangle+\left\langle\mu\left(G^{+} h_{0}\right), \eta-\eta_{0}\right\rangle+j(\xi) \\
\geq \int_{\Gamma}\left[\Psi_{N} \xi_{N}+\psi \cdot\left(\eta-\eta_{0}\right)\right] d s .
\end{gathered}
$$

Again, substituting into (16) $2 \varphi_{T}-\xi$ instead of $\xi$ and $2 \eta_{0}-\eta$ instead of $\eta$, we finally obtain

$$
\left|\int_{\Gamma}\left[\Psi_{N} \xi_{N}+\psi \cdot\left(\eta-\eta_{0}\right)\right] d s-\left\langle\sigma\left(G^{+} h_{0}\right), \xi-\varphi_{T}\right\rangle-\left\langle\mu\left(G^{+} h_{0}\right), \eta-\eta_{0}\right\rangle\right| \leq j(\xi) .
$$


Let now $\xi-\varphi_{T}=c$ and $\eta-\eta_{0}=a$, where $c=[a \times x]+b, a$ and $b$ are arbitrary constant vectors from $\mathbb{R}^{3}$. Since $\left\langle\mathcal{N}\left(G^{+} h_{0}\right), \rho\right\rangle_{\Gamma}=0(\rho=(c ; a))$, from (17) it follows that

$$
\left|\int_{\Gamma}\left[\Psi_{N} c_{N}+\psi \cdot a\right] d s\right| \leq \int_{\Gamma} g\left|c_{T}\right| d s
$$

Thus if (14) has a solution, then (18) is fulfilled for each $\rho$ which is a solution of the equation $\left\langle L^{+} h, h\right\rangle=0$ in the class $\left(H^{1 / 2}(\Gamma)\right)^{6}$ (the space of solutions of this equation is denoted by $\mathcal{R}^{+}$).

Let us show that if (18) is fulfilled strictly, i.e.,

$$
\int_{\Gamma} g\left|c_{T}\right| d s-\left|\int_{\Gamma}\left[\Psi_{N} c_{N}+\psi \cdot a\right] d s\right|>0, \quad \forall \rho=(c, a) \in \mathcal{R}^{+}, \rho \neq 0,
$$

then (14) has a solution.

Since $\mathcal{R}^{+}$is finite-dimensional, from (19) it follows that the following inequality

$$
\int_{\Gamma} g\left|c_{T}\right| d s-\left|\int_{\Gamma}\left[\Psi_{N} c_{N}+\psi \cdot a\right] d s\right|>M\|\rho\|_{0, \Gamma}, \quad \forall \rho \in \mathcal{R}^{+}, \quad \rho \neq 0,
$$

is fulfilled for some positive integer $M$.

On the space $\left(H^{1 / 2}(\Gamma)\right)^{6}$ let us consider the functional

$$
J(h)=\frac{1}{2}\left\langle L^{+} h, h\right\rangle+j(\xi)-\int_{\Gamma}\left[\Psi_{N} \xi_{N}+\psi \cdot \eta\right] d s, \quad \forall h=(\xi, \eta) \in\left(H^{1 / 2}(\Gamma)\right)^{6},
$$

and assume that $P$ is the orthogonal projection operator defined above.

Taking into account (20) and property (III) of the operator $L^{+}$, after some simple transformations we obtain the following estimate from (21):

$$
J(h) \geq c_{1}\|P h\|_{1 / 2, \Gamma}^{2}-c_{2}\|P h\|_{1 / 2, \Gamma}+M\|\rho\|_{0, \Gamma}-c_{3},
$$

where $h=P h+\rho, \rho \in \mathcal{R}^{+}$and $c_{i}, i=1,2,3$, are some positive integers.

Hence, passing to the limit as $\|h\|_{1 / 2, \Gamma} \longrightarrow \infty$, we conclude that $J(h) \longrightarrow$ $+\infty$, which proves that the functional $J(h)$ is coercive (it should be noted that if $\|h\|_{1 / 2, \Gamma} \longrightarrow \infty$, then for $\|P h\|_{1 / 2, \Gamma} \longrightarrow \infty$ we clearly have $J(h) \longrightarrow+\infty$, while for $\|\rho\|_{1 / 2, \Gamma} \longrightarrow \infty$ it readily follows that $\|\rho\|_{0, \Gamma} \longrightarrow \infty$ and therefore $J(h) \longrightarrow+\infty)$. We eventually obtain

Theorem 3. Let $\Gamma_{1}=\varnothing$ and (19) be fulfilled. Then under the conditions of Theorem 2 there exists a solution of Problem $(\Psi)^{+}$. Two arbitrary solutions of Problem $(\Psi)^{+}$differ in the rigid displacement vector. 


\section{EXTERIOR PROBlems}

As different from the interior problems, in this case we consider the homogeneous an isotropic exterior domain $\Omega^{-}$and, for the sake of simplicity, investigate a problem with friction in the classical elasticity theory.

Let

$$
A(\partial)=\left\|A_{j k}(\partial)\right\|_{3 \times 3}, \quad A_{j k}(\partial)=a_{i j e k} \frac{\partial^{2}}{\partial x_{i} \partial x_{e}}
$$

be a matrix differential operator of elastostatics, and

$$
T(\partial, \nu)=\left\|T_{j k}(\partial, \nu)\right\|_{3 \times 3}, \quad T_{j k}(\partial, \nu)=a_{i j e k} \nu_{i} \frac{\partial}{\partial x_{e}},
$$

be a stress operator $([7]), u=\left(u_{1}, u_{2}, u_{3}\right)$ be a displacement vector, and $a_{i j e k}$ be elastic constants which satisfy the conditions

$$
a_{i j e k}=a_{e k i j}=a_{j i e k}
$$

and $\exists \alpha_{0}>0$ such that $\forall \varepsilon_{i j} \in \mathbb{R}\left(\varepsilon_{i j}=\varepsilon_{j i}\right)$ we obtain

$$
a_{i j e k} \varepsilon_{i j} \varepsilon_{e k} \geq \alpha_{0} \varepsilon_{i j} \varepsilon_{i j}
$$

If $a_{i j e k}=\lambda \delta_{i j} \delta_{e k}+\mu\left(\delta_{i e} \delta_{j k}+\delta_{i k} \delta_{j e}\right)$, where $\lambda$ and $\mu$ are the Lameé constants, then we have an isotropic medium.

The bilinear form corresponding to this case has the form

$$
a(u, v)=a_{i j e k} \int_{\Omega^{-}} \varepsilon_{i j}(u) \varepsilon_{e k}(v) d x \quad\left(\varepsilon_{i j}(u)=\frac{1}{2}\left(\frac{\partial u_{i}}{\partial x_{j}}+\frac{\partial u_{j}}{\partial x_{i}}\right)\right)
$$

for every $u, v \in\left(H_{\text {loc }}^{1}\left(\Omega^{-}\right)\right)^{3}$ which in the neighborhood of infinity satisfy the conditions $u, v=O\left(|x|^{-1}\right)$ and $\frac{\partial u_{i}}{\partial x_{j}}, \frac{\partial v_{i}}{\partial x_{j}}=O\left(|x|^{-2}\right)$.

A nonhomogeneous problem with friction, analogous to Problem $(I)^{+}$, can be reduced, as we have done above, to

Problem $(\mathbf{F})^{-}$. Find a vector function $u \in\left(H_{\text {loc }}^{1}\left(\Omega^{-}\right)\right)^{3}$ which is a weak solution of the equation

$$
A(\partial) u(x)=0, \quad x \in \Omega^{-}
$$

in the neighborhood of infinity $u=O\left(\frac{1}{|x|}\right)$ and satisfies the conditions ${ }^{1}$

$$
\begin{aligned}
& \left.\sigma_{T}(u)\right|_{\Gamma_{2}} \in\left(L^{\infty}\left(\Gamma_{2}\right)\right)^{3},\left.u\right|_{\Gamma_{1}}=0,\left.\sigma_{N}(u)\right|_{\Gamma_{2}}=F_{N}, \text { and if }\left|\sigma_{T}(u)\right|<g \text {, then } \\
& u_{T}=\varphi_{T} \text { on } \Gamma_{2} \text {, and if }\left|\sigma_{T}(u)\right|_{\Gamma_{2}}=g \text {, then } \exists \lambda \geq 0: u_{T}=\varphi_{T}-\lambda \sigma_{T}(u), \\
& \text { where } \sigma(u)=T(\partial, \nu) u(x), \varphi \in\left(H^{1 / 2}\left(\Gamma_{2}\right)\right)^{3}, F_{N} \in L^{\infty}\left(\Gamma_{2}\right) \text { and } g= \\
& \mathcal{F}\left|F_{N}\right| \text {. }
\end{aligned}
$$

To investigate this problem, let us construct an operator $L^{-}$analogous to the operator $L^{+}$. To this end, we are to find a vector function $u \in\left(H_{\text {loc }}^{1}\left(\Omega^{-}\right)\right)^{3}$ vanishing at infinity and satisfying the condition $\left.u\right|_{\Gamma}=h, h \in\left(H^{1 / 2}(\Gamma)\right)^{3}$. As

\footnotetext{
1 The condition $\partial u_{i} / \partial x_{j}=O\left(|x|^{-2}\right)$ is fulfilled automatically (see [11]).
} 
is known, this problem has a unique solution which is given in the form of a simple-layer potential

$$
u(x)=\int_{\Gamma} \mathcal{T}(x-y)\left(\mathcal{H}^{-1}(h)\right)(y) d_{y} S, \quad x \in \Omega^{-},
$$

where $\mathcal{T}$ is a fundamental solution of the differential operator $A(\partial)([7])$, while the operator $\mathcal{H}$ is a value of the single-layer potential on the boundary $\Gamma$ :

$$
\mathcal{H}(h)(x)=\lim _{\Omega^{-} \ni z \rightarrow x \in \Gamma} \int_{\Gamma} \mathcal{T}(z-y) h(y) d_{y} S
$$

Note that $([12])$

$$
\begin{aligned}
& \mathcal{H}:\left(H^{s}(\Gamma)\right)^{3} \longrightarrow\left(H^{s+1}(\Gamma)\right)^{3}, \\
& \mathcal{H}^{-1}:\left(H^{s}(\Gamma)\right)^{3} \longrightarrow\left(H^{s-1}(\Gamma)\right)^{3}, \quad s \in \mathbb{R} .
\end{aligned}
$$

It is clear that for the first exterior problem the Green operator $G^{-}$is defined by formula (23), i.e.,

$$
\begin{gathered}
\forall h \in\left(H^{1 / 2}(\Gamma)\right)^{3}: A(\partial)\left(G^{-} h\right)(x)=0, \quad x \in \Omega^{-}, \\
\left.G^{-} h\right|_{\Gamma}=h, \quad G^{-} h=O\left(\frac{1}{|x|}\right) .
\end{gathered}
$$

The operator $L^{-}$is defined by the formula

$$
L^{-} h=\left\{\sigma\left(G^{-} h\right)\right\}_{\Gamma}^{-}, \quad \forall h \in\left(H^{1 / 2}(\Gamma)\right)^{3} .
$$

Taking into account the properties of the operator $G^{-}$, from the Green formula we obtain

$\forall h, g \in\left(H^{1 / 2}(\Gamma)\right)^{3}:\left\langle L^{-} h, g\right\rangle=a\left(G^{-} h, G^{-} g\right)=a_{i j e k} \int_{\Omega^{-}} \varepsilon_{i j}\left(G^{-} h\right) \varepsilon_{e k}\left(G^{-} g\right) d x$.

The operator $L^{-}$is a strongly elliptic self-conjugate invertible pseudodifferential operator of first order on the closed manifold $\Gamma$, having the following properties:

(I) $\left\langle L^{-} h, g\right\rangle=\left\langle L^{-} g, h\right\rangle, \forall h, g \in\left(H^{1 / 2}(\Gamma)\right)^{3}$;

(II) $L^{-}:\left(H^{1 / 2}(\Gamma)\right)^{3} \longrightarrow\left(H^{-1 / 2}(\Gamma)\right)^{3}$ is a continuous mapping;

(III) $\exists c>0:\left\langle L^{-} h, h\right\rangle \geq c\|h\|_{1 / 2, \Gamma}^{2}, \forall h \in\left(H^{1 / 2}(\Gamma)\right)^{3}$.

Properties (I) and (II) are trivial to prove. As to property (III) of operator $L$, it immediately follows from a more general result presented in the monograph by Mclean (see [13], Ch. II).

Analogously to the preceding case, Problem $(F)^{-}$is equivalently reduced to the variational inequality:

Find $h_{0} \in K$ such that $\forall h \in K$

$$
\left\langle L^{-} h_{0}, h-h_{0}\right\rangle+j(h)-j\left(h_{0}\right) \geq \int_{\Gamma_{2}} F_{N}\left(h_{N}-h_{0 N}\right) d s
$$


where $K=\left\{h \in\left(H^{1 / 2}(\Gamma)\right)^{3}:\left.h\right|_{\Gamma_{1}}=0\right\}$ and $j(h)=\int_{\Gamma_{2}} g\left|h_{T}-\varphi_{T}\right| d s$.

The investigation of the existence and uniqueness of the solution (25) is mainly based on the properties of the operator $L^{-}$and it turns out that the corresponding functional

$$
\mathcal{J}(h)=\frac{1}{2}\left\langle L^{-} g, h\right\rangle+j(h)-\int_{\Gamma_{2}} F_{N} h_{N} d s
$$

is coercive on the space $\left(H^{1 / 2}(\Gamma)\right)^{3}$ regardless whether $\Gamma_{1}$ has a positive measure or is empty. We eventually arrive at

Theorem 4. If $F_{N} \in L^{\infty}\left(\Gamma_{2}\right)$ and $\varphi \in\left(H^{1 / 2}(\Gamma)\right)^{3}$, then Problem $(F)^{-}$has a unique solution.

The obtained theorem also remains valid in the case where $\Gamma_{1}=\varnothing\left(\Gamma_{2}=\Gamma\right)$.

\section{REFERENCES}

1. G. Duvaut and J.-L. Lions, Les inéquations en mécanique et en physique. Travaux et Recherches Math'ematiques, No. 21. Dunod, Paris, 1972; Russian transl.: Nauka, Moscow, 1980.

2. I. HlavaČEK, J. Haslinger, J. NeČAs, and J. LoviŠEK, Solution of variational inequalities in mechanics. (Slovak) Alfa-Vydavatel'stvo Technickej a Ekonomickej Literatúry, Bratislava; Státní Nakladatelství Technické Literatury (SNTL), Prague, 1982; Russian transl.: Mir, Moscow, 1986.

3. J. JARUŠEK, Contact problems with bounded friction coercive case. Czechoslovak Math. J. 33(108)(1983), No. 2, 237-261.

4. R. L. Gachechiladze and O. I. Maisaia, Some boundary value problems of the couplestress elasticity with frictions. Proc. A. Razmadze Math. Inst. 119(1999), 25-41.

5. R. Gachechiladze, Signorini's problem with friction for a layer in the couple-stress elasticity. Proc. A. Razmadze Math. Inst. 122(2000), 45-57 (http://www.rmi.acnet.ge/proceedings).

6. J. NEČAS, J. JARUŠEK, and J. HASLINGER, On the solution of the variational inequality to the Signorini problem with small friction. Boll. Un. Mat. Ital. B (5) 17(1980), No. 2, 796-811.

7. V. D. Kupradze, T. G. Gegelia, M.O. Basheleishvili, and T. V. Burchuladze, Three-dimensional problems of the mathematical theory of elasticity and thermoelasticity. (Translated from the Russian) North-Holland Series in Applied Mathematics and Mechanics, 25. North-Holland Publishing Co., Amsterdam-New York, 1979; Russian original: Nauka, Moscow, 1976.

8. J.-L. Lions and E. Magenes, Problèmes aux limites non homogènes et applications. Vol. 1, 2. Travaux et Recherches Mathématiques, No. 17, 18. Dunod, Paris, 1968.

9. J. L. Lions, Quelques méthodes de résolution des problèmes aux limites non linéaires. Dunod; Gauthier-Villars, Paris, 1969.

10. A. Fridman, Variational principles and free-boundary problems. A Wiley-Interscience Publication. Pure and Applied Mathematics. John Wiley \& Sons, Inc., New York, 1982.

11. T. V. Buchukuri and T. G. Gegelia, Boundary value problems in elasticity theory with concentrated singularities. (Russian) Differentsial'nye Uravneniya 25(1989), No. 10, 1746-1755; English transl.: Differential Equations 25(1989), No. 10, 1226-1234 (1990). 
12. A. Gachechiladze and D. Natroshvili, Boundary variational inequality approach in the anisotropic elasticity for the Signorini problem. Georgian Math. J. 8(2001), No. 3, 469-492.

13. W. McLean, Strongly elliptic systems and boundary integral equations. Cambridge University Press, Cambridge, 2000.

(Received 20.02.2003; revised 13.09.2004)

Author's address:

A. Razmadze Mathematical Institute

Georgian Academy of Sciences

1, M. Aleksidze St., Tbilisi 0193

Georgia 